\title{
Role of Intraoperative ICP And CPP Measurement for Predicting Surgical Outcome in Severe Traumatic Brain Injury
}

\author{
Ramesh Chandra VV ${ }^{1}$ Chandra Mowliswara Prasad Bodapati ${ }^{1} \quad$ Rajesh Paradesi ${ }^{1}$ \\ ${ }^{1}$ Department of Neurosurgery, Sri Venkateswara Institute of \\ Medical Sciences (SVIMS), Tirupati, Andhra Pradesh, India

\begin{abstract}
Address for correspondence Rajesh Paradesi, MS, MCh, Department of Neurosurgery, Sri Venkateswara Institute of Medical Sciences (SVIMS), Tirupati 517507, Andhra Pradesh, India (e-mail: rajeshparadesh@gmail.com).
\end{abstract}

Indian J Neurotrauma:2020;17:121-129

\begin{abstract}
Introduction Traumatic brain injury (TBI) is one of the leading causes of mortality and disability worldwide, and optimizing the management of these patients is a continuing challenge. Intraoperative intracranial pressure (ICP) and cerebral perfusion pressure (CPP) were evaluated for use as prognostic indicators after surgery for severe TBI. Although ICP and CPP monitoring is standard postsurgery treatment for TBI, very few studies have reported the use of ICP and CPP values monitored during surgery.

Objectives The objectives of this study were to evaluate the use of intraoperative ICP and CPP values as prognostic indicators and as subjective guidelines for managing severe TBI.

Materials and Methods All patients with severe TBI who underwent surgical decompression and ICP monitoring intraoperatively were included in our study from 2017 to 2018. We measured ICP and CPP values after creation of the first burr hole, after hematoma evacuation, and after wound closure.

Keywords

- intracranial pressure

- cerebral perfusion pressure

- Glasgow outcome scale

- receiver-operating characteristic

Results From the analysis of receiver-operated characteristic (ROC) curves, we observed that ICP initial (cutoff $>28 \mathrm{~mm} \mathrm{Hg}$ ) and CPP initial (cutoff $<44.5 \mathrm{~mm} \mathrm{Hg}$ ) are the best predictors of unfavorable outcomes. Favorable outcome (Glasgow outcome scale [GOS] 4 and 5) and unfavorable outcome (GOS 1-3) after 6 months were achieved in 64.1 and $35.8 \%$ of patients, respectively. There was significant difference between the ICP and CPP values which are measured after the first burrhole, after hematoma evacuation, and after scalp closure in both favorable and unfavorable outcomes. The highest positive Pearson's correlation coefficient is found between GOS and ICP and CPP after first burr hole.

Conclusion Monitoring ICP and CPP during surgery improves management in patients with severe TBI and provides an early prognostic indicator in such patients.
\end{abstract}

\section{Introduction}

Traumatic brain injury (TBI), a huge clinical challenge and public health problem, is one of the leading causes of mortality and disability worldwide, ${ }^{1}$ and optimizing the management of these patients is a continuing challenge. Mortality and disability in patients with TBI are the consequence of the primary injury, as well as secondary damage resulting from increased intracranial pressure (ICP) due to traumatic mass lesions, increasing hematoma, and brain edema or swelling. ${ }^{2}$ The ICP monitor provides neurosurgeons with early and sufficiently precise information for detecting intracranial lesions. The insertion of an ICP monitor has proven effective
DOI https://doi.org/

$10.1055 / \mathrm{s}-0040-1713324$

ISSN 0973-0508.
(C)2020 Neurotrauma Society of India
License terms

()(1) $\Theta \circledast$ 
for improving surgical outcomes in severe TBI. Information provided by ICP monitoring is also used in targeted therapies that are now standard treatment for severe TBI., ${ }^{3,4}$

Many parameters can be used to monitor the status of head-injured patients. Of the ones most commonly used, the most important appear to be ICP and cerebral perfusion pressure (СРP). Since the landmark paper by Lundberg, ${ }^{5}$ in which the clinical use of ICP monitoring was first demonstrated, it has become a standard procedure in the management of severely head-injured patients. There exists a large body of work on both the methods of measurement and their usefulness in clinical management. However, until the prospective randomized controlled trial of Robertson et $\mathrm{al},{ }^{6}$ there had been little published evidence on the precise determination of threshold values of ICP and CPP or comparisons regarding which of these variables is more important in determining the outcome.

Although ICP and CPP monitoring is the standard postsurgery treatment for TBI, very few studies have reported the use of ICP and CPP values monitored during surgery. A study of outcomes after surgery for severe $\mathrm{TBI}^{7}$ showed that patients whose surgical treatment had included aggressive ICP monitoring had significantly better outcomes compared with patients who had not received ICP monitoring during surgery.

In this study, we evaluate the use of intraoperative ICP and CPP values as prognostic indicators and as subjective guidelines for managing severe TBI.

\section{Aims and Objective}

- Measurement of ICP and CPP.

- To predict the surgical outcome in severe TBI based on intraoperative ICP and CPP.

\section{Materials and Methods}

\section{Patient Selection}

All patients with severe TBI presenting to the Department of Neurosurgery at SVIMS, Tirupati, from April 2017 to July 2018 were included. The inclusion criteria were patients who had sustained severe TBI, which was defined as a head injury with a Glasgow coma scale (GCS) score $\leq 8$, and patients undergoing ICP monitoring during surgery. The exclusion criteria were patients not undergoing ICP monitoring during surgery, patients with metabolic or drug-related unconscious status, patients with polytrauma, and patients who met the inclusion criteria and lost to follow-up after discharge.

In all of the above patients, a detailed medical history was obtained and thorough clinical testing was conducted. Patients underwent a brain CT scan (SIEMENS 128 slice, Germany 2009) to establish the diagnosis. The clinical characteristics, GCS score, trauma mechanism, cerebral hemorrhage type, and Glasgow outcome scale (GOS) score at 6 months after injury were studied and the details were recorded on a structured proforma.

\section{Surgery}

An appropriate surgery was planned based on the radiological findings. The Codman ICP monitoring system (Codman ICP Micro Sensor; Johnson \& Johnson, Raynham, MA) measures intracranial pressure at the parenchymal level. When making the trauma flap, the burr holes were created along the path of the trauma flap and over the Kocher point to avoid the motor strip. The microsensor was inserted in the parenchyma. In patients who underwent surgical decompression and ICP monitoring during the surgical procedure, the data analysis included ICP and CPP values after the creation of the first burr hole, after hematoma evacuation, and after wound closure. CPP is defined as the difference between the mean arterial pressure (MAP) and ICP (CPP $=$ MAP-ICP). $\mathrm{MAP}=$ diastolic blood pressure $(\mathrm{BP})+1 / 3$ pulse pressure $(\mathrm{PP})$.

\section{Statistical Analysis}

All the data was tabulated in a Microsoft Excel 2007 datasheet under various headings. All the data was expressed as mean and standard deviation. The ICP ( $\mathrm{mm} \mathrm{Hg}$ ) and $\mathrm{CPP}(\mathrm{mm} \mathrm{Hg})$ values measured after the creation of the first burr hole, after hematoma evacuation, and after wound closure will be compared by using receiver-operating characteristic (ROC) curves. Outcomes were determined 6 months after head injury, with a favorable outcome defined as a GOS of 4 to 5 (moderate disability or better) and an unfavorable outcome defined as a GOS of 1 to 3 (death, vegetative state, or severe disability). The relationships between ICP and CPP values and GOS scores will be evaluated by correlation analysis. A $p$-value $<0.05$ will be considered statistically significant. The statistical package for social sciences (SPSS) version 20.0 software will be used for all statistical analyses.

\section{Observation and Results}

In total, 53 severe TBI patients were prospectively evaluated. Road traffic accidents occurred in $79.2 \%$ of cases and accidental falls occurred in $20.7 \%$ of cases. The mean age of the 53 patients ( 40 male and 13 female) was $43.7 \pm 15.84$ years (range of 20-61 years). There was a significant predominance of male patients (66.7\%). Subdural hemorrhages were present in $34.1 \%$ of cases, intracranial contusion hematomas in $22.6 \%$, and mixed hemorrhages in $43.3 \%$. The mean preoperative GCS score was $5.39 \pm 1$. At 6 months postoperatively, $64.1 \%$ (34/53) of patients had favorable outcomes, with a GOS of 4 to $5 ; 35.8 \%$ (19/53) of patients had unfavorable outcomes, with a GOS of 1 to 3 .

-Figs. 1-3 show the ROC curves for ICP and CPP at different stages of the operation (after the creation of the first burr hole, after the evacuation of hematoma, and after scalp closure). The largest areas under the ICP and CPP ROC curve were $(1,0.8,0.88),(0.944,0.944,0.889)$ after the first burr hole creation, after the evacuation of hematoma, and after scalp closure, respectively.In the majority of cases, ICP had the greatest AUC during the different stages of surgery. At a value of $\geq 28 \mathrm{~mm} \mathrm{Hg}$, the sensitivity for ICP after the creation of the 

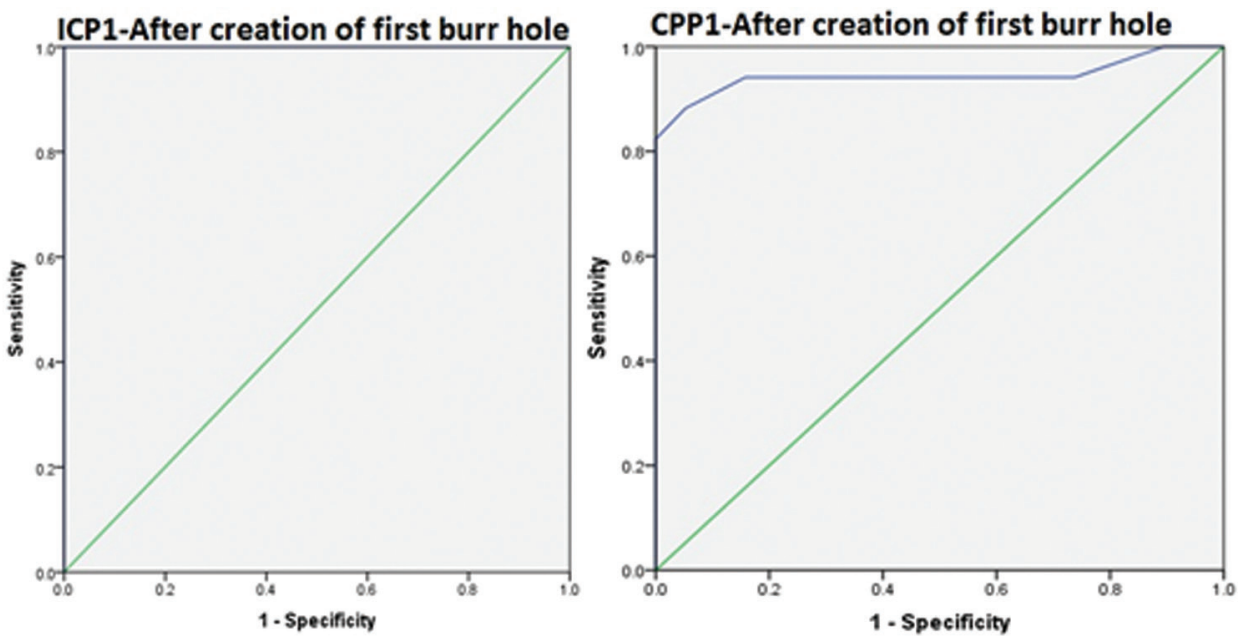

Fig. 1 ROC curves of ICP (left) and CPP (right) after the creation of the first burr hole. CPP, cerebral perfusion pressure; ICP, intracranial pressure; ROC, receiver-operating characteristic.
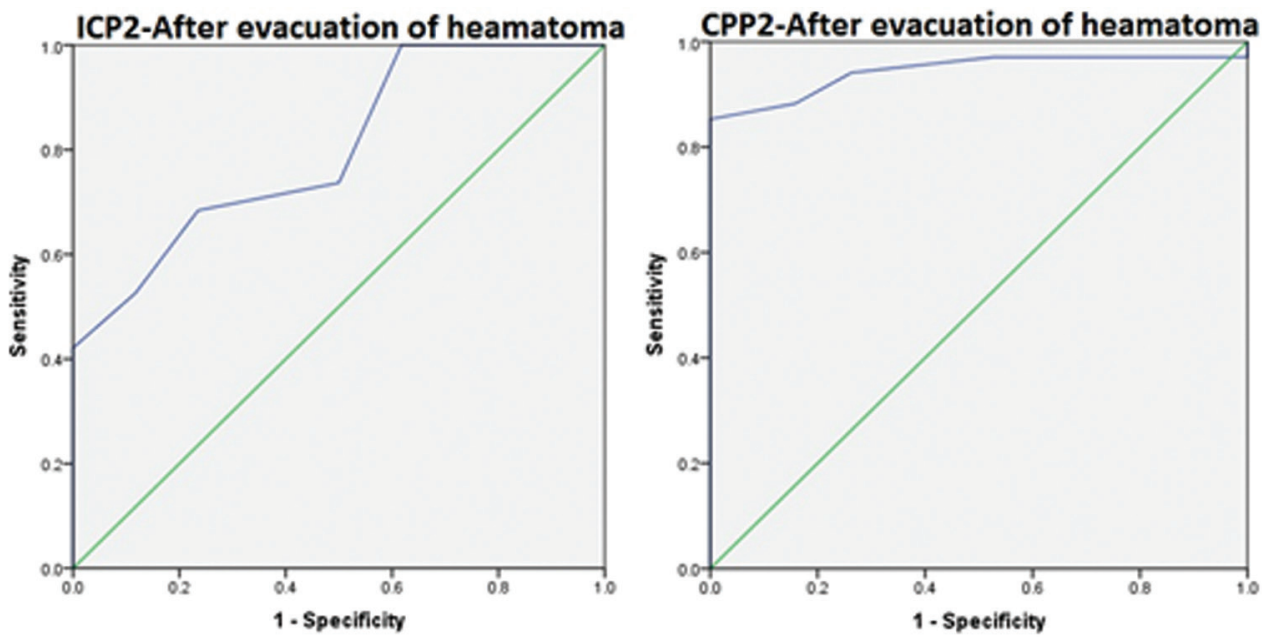

Fig. 2 ROC curves of ICP (left) and CPP (right) after the evacuation of the hematoma. CPP, cerebral perfusion pressure; ICP, intracranial pressure; ROC, receiver-operating characteristic.
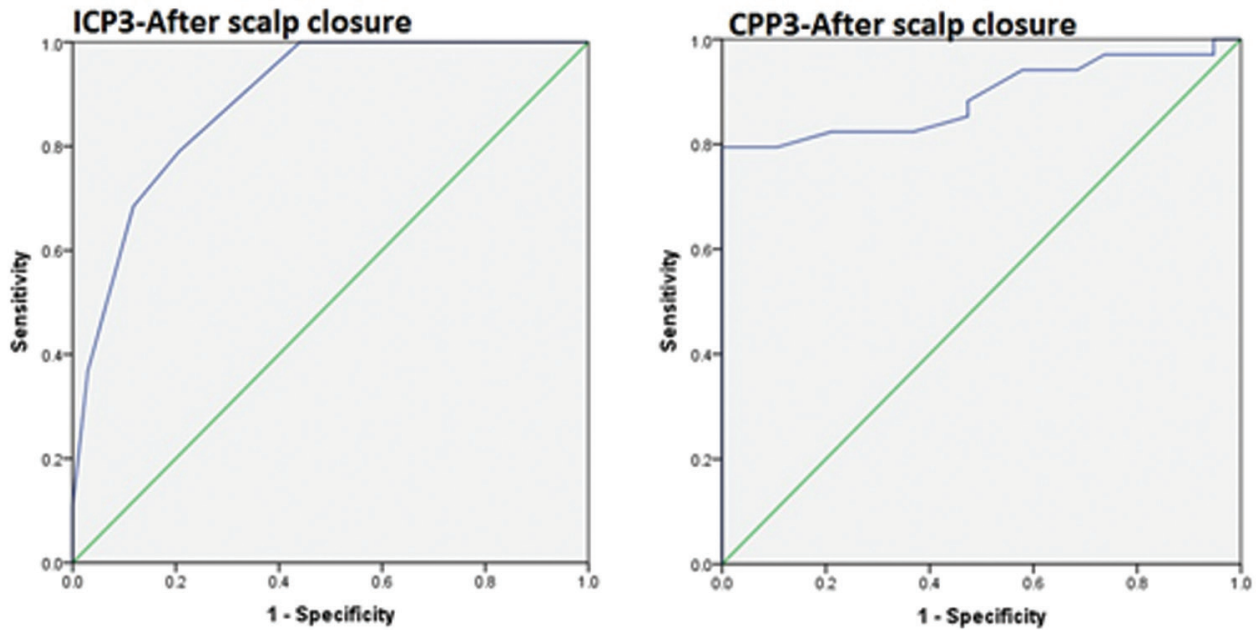

Fig. 3 ROC curves of ICP (left) and CPP (right) after scalp closure. CPP, cerebral perfusion pressure; ICP, intracranial pressure; ROC, receiver-operating characteristic. 
first burr hole was 1; patients with an average ICP after the creation of the first burr hole of more than or equal to $28 \mathrm{~mm}$ $\mathrm{Hg}$ had unfavorable outcomes. At a value of $44.5 \mathrm{~mm} \mathrm{Hg}$, the sensitivity for CPP after the creation of the first burr hole was 0.882; patients with an average CPP after the creation of the first burr hole of less than or equal to $44.5 \mathrm{~mm} \mathrm{Hg}$ had unfavorable outcomes.

The ROC curve for ICP after the creation of the first burr hole at false-positive rate (1-specificity) 0 and at $28 \mathrm{~mm}$ $\mathrm{Hg}$ to a value of 1.0 at $18 \mathrm{~mm} \mathrm{Hg}$ indicated that patients with ICP of greater than or equal to $28 \mathrm{~mm} \mathrm{Hg}$ had an unfavorable outcome, and patients between 18 to $28 \mathrm{~mm} \mathrm{Hg}$ had a favorable outcome. At a value of $28 \mathrm{~mm} \mathrm{Hg}$, the sensitivity was 1 and the false positive rate (1-specificity) was 0 . At this point, the curve was farthest from the diagonal.

ROC curve for CPP after the creation of the first burr hole at false-positive rate (1-specificity) 0 and at $45.5 \mathrm{~mm} \mathrm{Hg}$ to a value of 1.0 at $28 \mathrm{~mm} \mathrm{Hg}$ indicated that patients with CPP of greater than or equal to $45.5 \mathrm{~mm} \mathrm{Hg}$ had a favorable outcome, and patients between 28 to $45.5 \mathrm{~mm} \mathrm{Hg}$ had an unfavorable outcome.

From - Table 1, which shows the analysis of ROC curves, we observe that ICP initial (cutoff $>28 \mathrm{~mm} \mathrm{Hg}$ ) and CPP initial (cutoff $<44.5 \mathrm{~mm} \mathrm{Hg}$ ) are the best predictors of unfavorable outcomes.

- Fig. 4 shows the differences in ICP and CPP between the creation of the first burr hole and hematoma evacuation, between hematoma evacuation and wound closure, and between creation of the first burr hole and wound closure, which were as follows: $18.3 \pm 8.31 \mathrm{~mm} \mathrm{Hg}$, $5.41 \pm 2.59 \mathrm{~mm} \mathrm{Hg}$, and $8.6 \pm 2.69 \mathrm{~mm} \mathrm{Hg} ; 19.89 \pm 10.91 \mathrm{~mm} \mathrm{Hg}$, $4.89 \pm 9.7 \mathrm{~mm} \mathrm{Hg}$, and $24.78 \pm 6.4 \mathrm{~mm} \mathrm{Hg}$, respectively.

- Table 2 shows there was a significant difference between the ICP and CPP values which are measured after the first burr hole, after hematoma evacuation, and after scalp closure in both favorable and unfavorable outcomes.

- Table 3 shows the Pearson's correlation coefficient between GOS and other variables (ICP and CPP). All the correlations are significant. The highest positive correlation is found between GOS, ICP and CPP after the first burr hole.

- Table 4 shows favorable outcome (GOS score of 1 to 3 ) after 6 months, which was achieved in $64.1 \%$ (34/53) of patients; unfavorable outcome (GOS of 1 to 3 ), which was achieved in $35.8 \%(19 / 53)$ of the patients.

\section{Codman ICP Monitor and Intraoperative Images}

-Fig. 5: Codman ICP monitor, microtransducer.
- Fig. 6: Intraoperative ICP measurement steps-after first burr hole, after hematoma evacuation, and after scalp closure.

\section{Discussion}

ICP monitoring is currently considered the standard of care for patients with severe TBI which provides the ability to monitor ICP, CPP, or tissue-perfusion modification. ${ }^{8}$

The early values of ICP and CPP during neurosurgery for severe head injury are not well-studied. This is primarily because the surgical team is concerned with the rapid removal of the hematoma, rather than placement of monitoring devices before clot evacuation. Of the ICP monitors placed intraoperatively, most are placed at the end of the surgery, and the first ICP measurement is not taken until after scalp closure or on arrival at the intensive care unit (ICU). However, the presence of an ICP monitor intraoperatively allows calculation of CPP (MAP-ICP), which may be important. Rosner and Daughton demonstrated the importance of maintaining adequate CPP in patients with head injuries at the ICU, and it seems prudent to do so in the operating room as well. ${ }^{9}$

The effects of decompressive craniectomy in patients with intracranial hypertension have been reported. ${ }^{10-13}$ However, most of these reports do not show the relationship between ICP and CPP during the course of the entire operation. Continuous monitoring of ICP and CPP may play key roles in the prognostic evaluation of severe TBI patients, and the appropriate therapeutic measures and nursing intervention on intracranial hypertension were conducive to decrease severe disability and mortality rates and improve the quality of life of patients. ${ }^{14}$ Another benefit of intraoperative monitoring of ICP and CPP during surgery for severe TBI involves predicting outcome. Very few studies have proposed intraoperative thresholds for ICP and CPP during surgery for severe TBI. In addition to this, in our study, the results of these analyses demonstrated significant differences in the relationships between ICP, CPP, and outcome at different stages of the operation.

In our study, the ROC curves showed that both ICP and CPP are better predictors of outcome because the curves of ICP and CPP lie in similar positions and the areas under the curves are almost similar in the different stages of surgery ( 1 and 0.944 after the creation of the first burr hole, 0.80 and 0.944 after hematoma evacuation, and 0.80 and 0.88 after scalp closure). The largest area under the ICP and CPP ROC curves were 1 and 0.944 found after the creation of the the first burr hole, suggesting that the ICP and CPP after the

Table 1 Area under the ROC curve, and ICP and CCP thresholds during operative procedures for severe head injury $(n=53)$

\begin{tabular}{|l|l|l|l|l|}
\hline \multirow{2}{*}{ Observation time } & \multicolumn{2}{|c|}{ ICP } & \multicolumn{2}{c|}{ CPP } \\
\cline { 2 - 5 } & $\begin{array}{l}\text { Area under } \\
\text { ROC curve }\end{array}$ & $\begin{array}{l}\text { Threshold } \\
(\mathrm{mm} \mathrm{Hg})\end{array}$ & $\begin{array}{l}\text { Area under } \\
\text { ROC curve }\end{array}$ & $\begin{array}{l}\text { Threshold } \\
(\mathrm{mm} \mathrm{Hg})\end{array}$ \\
\hline After creation of first burr hole & 1 & 28 & 0.944 & 44.5 \\
\hline After the evacuation of hematoma & 0.800 & 10.5 & 0.944 & 62 \\
\hline After scalp closure & 0.888 & 15.5 & 0.889 & 69.5 \\
\hline
\end{tabular}

Abbreviations: CPP, cerebral perfusion pressure; ICP, intracranial pressure; ROC, receiver-operating characteristic. 
creation of the first burr hole possess the greatest accuracy for predicting outcome compared with other phases of the operative procedure.

Our results - Table 5 are comparable to Kuo et al, ${ }^{15}$ a study on intraoperative applications of ICP monitoring in patients with a severe head injury. In a study by Chambers et $a l,{ }^{16}$ it was shown that the largest area under the ICP and CPP ROC curves were 0.67 (CPPmin of $55 \mathrm{~mm} \mathrm{Hg}$ ) and 0.64 (ICPmax of $35 \mathrm{~mm} \mathrm{Hg}$ ), which appear to be the best predictors in adults (-Fig. 7).

Low et al reported that the combined use of physiological and biochemical variables improves predictive accuracy. ${ }^{17}$ Although some prognostic models for predicting surgical outcomes in severe TBI have been validated, no prognostic model has used ICP and CPP values obtained during and after surgery. In a study by Kuo et $\mathrm{a}^{15}$, the ROC curves showed that CPP was a better predictor of the outcome in

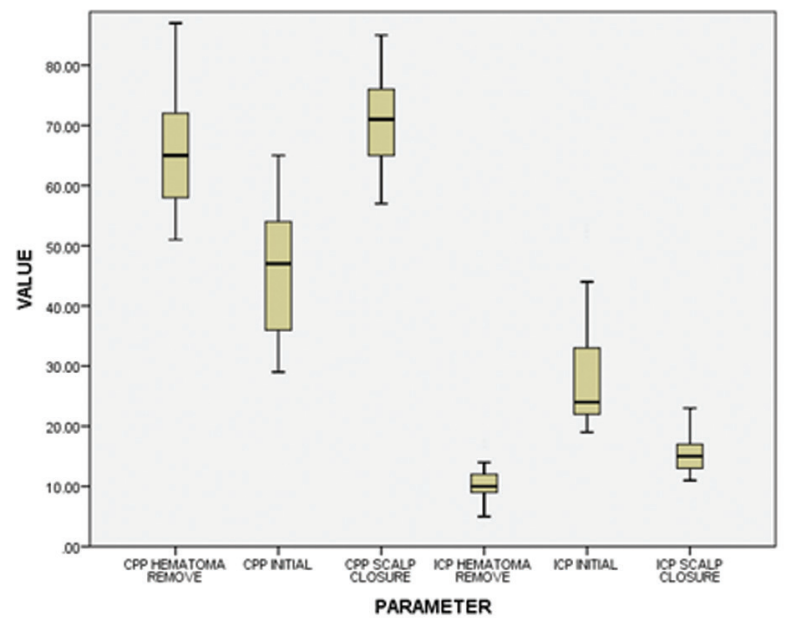

Fig. 4 Compares ICP and CPP values after the creation of the first burr hole after hematoma evacuation and after scalp closure. CPP, cerebral perfusion pressure; ICP, intracranial pressure. the majority of cases, because the curves lie above and to the left of the ICP, and the areas under the curves are greater in the different stages of surgery. This means that the CPP is more predictive of outcome than ICP at every stage of surgery. We contradicted their study, except after creation of the first burr hole, the curves of ICP and CPP lay similar, and the areas under the curves were almost similar in the different stages of surgery; thus, we concluded that both ICP and CPP are good predictors of outcome and need to be closely monitored.

Table 3 The relationship between the level of ICP and CPP, and the GOS in severe TBI

\begin{tabular}{|l|l|l|}
\hline Variable & $\mathbf{r}$ & $\boldsymbol{p}$-Value \\
\hline ICP-initial & 0.678 & $<0.0001$ \\
\hline ICP-hematoma removal & 0.235 & $<0.0002$ \\
\hline ICP-scalp closure & 0.407 & $<0.0001$ \\
\hline CPP-initial & 0.524 & $<0.0001$ \\
\hline CPP-hematoma remove & 0.383 & $<0.0001$ \\
\hline CPP-scalp closure & 0.300 & $<0.0001$ \\
\hline
\end{tabular}

Abbreviations: CPP, cerebral perfusion pressure; GOS, Glasgow outcome scale; ICP, intracranial pressure; TBI, traumatic brain injury.

Table 4 Outcome according to the GOS, expressed in percentage, after 6 months of injury

\begin{tabular}{|l|l|l|l|}
\hline GOS & Grade & Frequency & Percentage \\
\hline Death & 1 & 15 & $28 \%$ \\
\hline Vegetative state & 2 & 2 & $4 \%$ \\
\hline Severe disability & 3 & 2 & $4 \%$ \\
\hline Moderate disability & 4 & 10 & $19 \%$ \\
\hline Good recovery & 5 & 24 & $45 \%$ \\
\hline Total & & 53 & $100 \%$ \\
\hline
\end{tabular}

Abbreviation: GOS, Glasgow outcome scale.

Table 2 Comparison of patients with a severe head injury, and favorable and unfavorable outcomes

\begin{tabular}{|l|l|l|l|}
\hline Variable & $\begin{array}{l}\text { Favorable outcome }(\boldsymbol{n}=\mathbf{3 4}) \\
\text { Mean } \pm \text { SD }\end{array}$ & $\begin{array}{l}\text { Unfavorable outcome }(\boldsymbol{n}=19) \\
\text { Mean } \pm \text { SD }\end{array}$ & $p$-value \\
\hline Male $(\%)$ & 41.5 & 33.9 & \\
\hline Female $\%$ & 22.6 & 1.8 & \\
\hline Age & 46.21 & 41.26 & $<0.0001$ \\
\hline ICP1-after first burr hole creation & $22.88 \pm 1.85$ & $37.63 \pm 6.76$ & $<0.0001$ \\
\hline ICP2-after hematoma evacuation & $8.85 \pm 2.22$ & $11.68 \pm 2.21$ & $<0.0001$ \\
\hline ICP3-after scalp closure & $14 \pm 2.07$ & $17.58 \pm 2.09$ & $<0.0001$ \\
\hline CPP1-after first burr hole creation & $51.94 \pm 8.13$ & $34.74 \pm 4.69$ & $<0.0001$ \\
\hline CPP2-after hematoma evacuation & $70.62 \pm 8.57$ & $56.79 \pm 2.70$ & $<0.0001$ \\
\hline CPP3-after scalp closure & $73.59 \pm 5.92$ & $65.11 \pm 3.81$ & \\
\hline
\end{tabular}

Abbreviations: CPP, cerebral perfusion pressure; ICP, intracranial pressure; SD, standard deviation. 

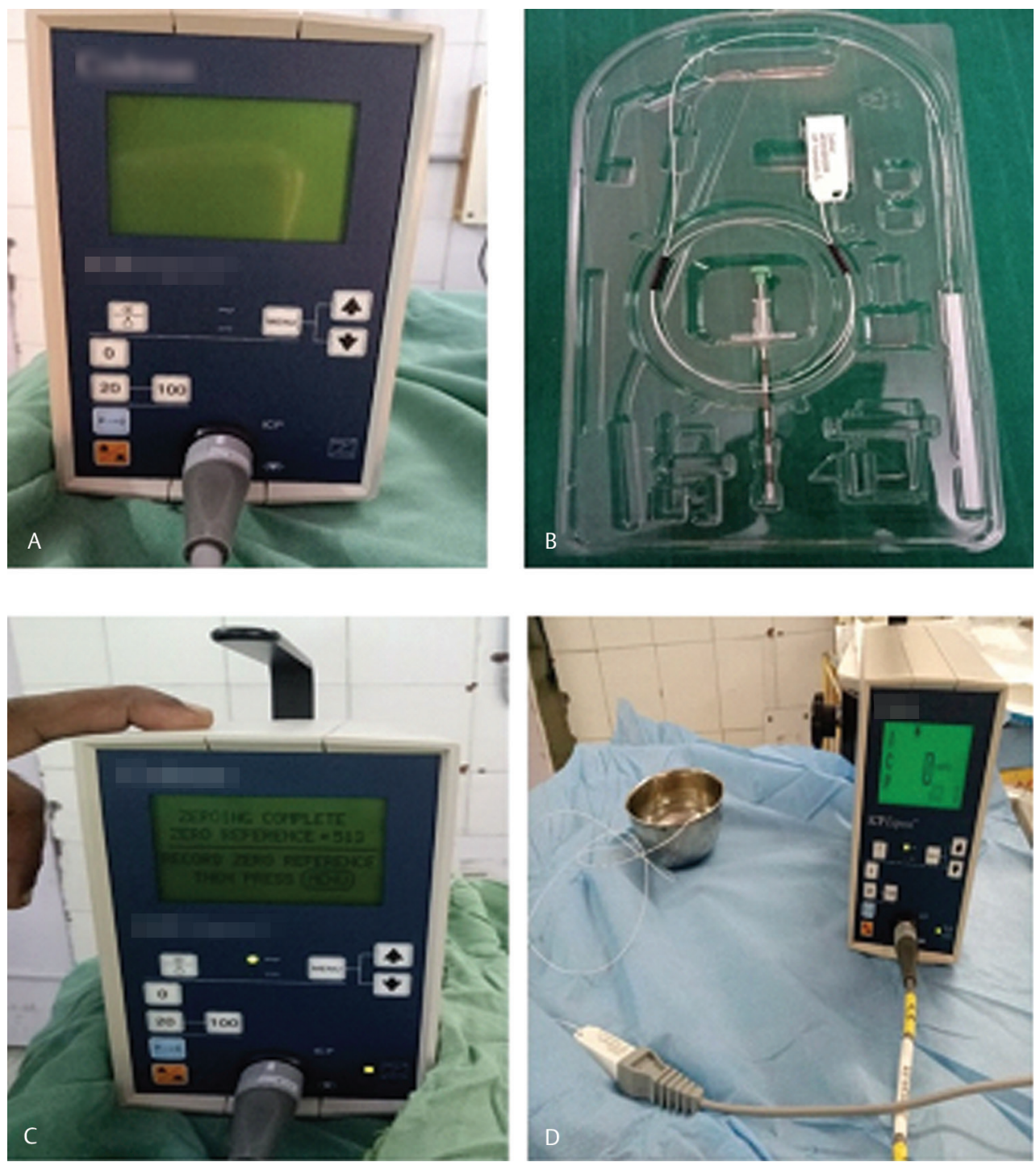

Fig. 5 (A-D): Codman ICP monitor, microtransducer. ICP, intracranial pressure.

The third edition of the Guidelines for the Management of Severe Traumatic Brain Injury (2007) recommends that surgery should be performed when the ICP threshold exceeds $20 \mathrm{~mm} \mathrm{Hg}$ or when the CPP range is from 50 to $70 \mathrm{~mm} \mathrm{Hg}$. Surgery should be avoided when CPP is $<50 \mathrm{~mm} \mathrm{Hg.}{ }^{18}$ Very few studies have proposed intraoperative thresholds for ICP and CPP during surgery for severe TBI. Based on the data obtained in this study, critical thresholds ([28 and $44.5 \mathrm{~mm} \mathrm{Hg}]$, [10.5 and $62 \mathrm{~mm} \mathrm{Hg}$ ], and [15.5 and $69.5 \mathrm{~mm} \mathrm{Hg}$ ]) are proposed for ICP and CPP measured after the first burr hole creation, after hematoma evacuation, and after wound closure, respectively. Our results are comparable to Tsai et al who in their study proposed the critical thresholds ([14 and $56 \mathrm{~mm} \mathrm{Hg}$ ] and [20 and $50 \mathrm{~mm} \mathrm{Hg}$ ]) for ICP and CPP measured after hematoma evacuation and after wound closure, respectively. ${ }^{19}$ Our results also almost similar to the Kuo et. al, who in their study proposed the critical thresholds ([17.5 and $51.8 \mathrm{~mm} \mathrm{Hg}$ ] and [22 and $52 \mathrm{~mm} \mathrm{Hg}$ ]) for ICP and CPP measured after hematoma evacuation and wound closure, respectively. ${ }^{15}$
To the best of our knowledge, our study is the one of the few studies to evaluate the correlation between intraoperative ICP and CPP. In our study, we observed that the initial ICP $(28.17 \pm 8.31 \mathrm{~mm} \mathrm{Hg})$ is significantly higher and the CPP (45.77 \pm 10.91$)$ is significantly lower in all patients with both favorable and unfavorable outcomes. The ICP $(9.87 \pm 2.59 \mathrm{~mm} \mathrm{Hg})$ decreased immediately after removal of the bone flap and decreased further after opening of the dura and removal of the blood clot. Simultaneously, the values of CPP (65.66 $\pm 9.7 \mathrm{~mm} \mathrm{Hg})$ increased stepwise in patients with both favorable and unfavorable outcomes. Our results are also comparable to Tsai et al who in their study found the values of ICP $(33.9 \pm 11.1,10.8 \pm 8.9,17.6 \pm 11.4 \mathrm{~mm} \mathrm{Hg})$ and $\mathrm{CPP}(45.8 \pm 11.4,66.7 \pm 10.6,60.5 \pm 10.7 \mathrm{~mm} \mathrm{Hg})$ after the creation of the first burr hole, after hematoma evacuation, and after wound closure respectively. ${ }^{17}$

In our study, we measured the outcome with the GOS scale. The GOS is the most widely used outcome measure after TBI. Studies have suggested that assessment of the GOS using a standard format with a written protocol is practical and reliable. ${ }^{20}$ 

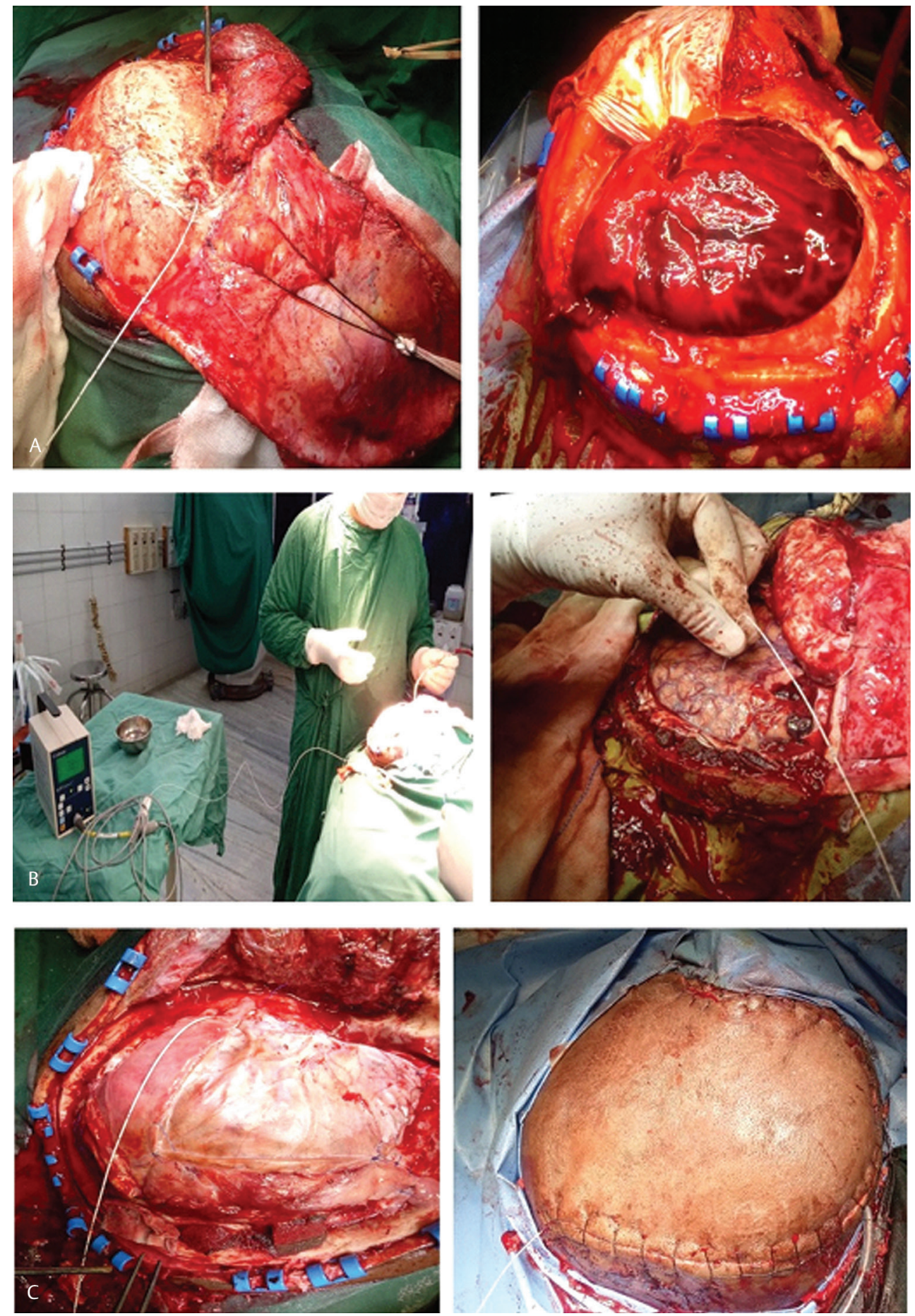

Fig. 6 Intraoperative ICP measurement steps after first burr hole (A), after hematoma evacuation (B), and after scalp closure (C). CPP, cerebral perfusion pressure; ICP, intracranial pressure.

This is the first study attempting to quantify the association of intraoperative ICP and CPP values with GOS. In the entire study population, the overall mortality was $28 \%$ (15/53), $36 \%$ (19/53) had poor neurological outcome (severely disabled [SD] or vegetative [V]), and 64\% (34/53) had good recovery (GR) or moderate disability (MD). Patients with an ICP $<28 \mathrm{~mm} \mathrm{Hg}$ and CPP $>45.5 \mathrm{~mm} \mathrm{Hg}$ presented GR/MD in $64 \%$ of the cases, Patients with an ICP $>28 \mathrm{~mm} \mathrm{Hg}$ and $\mathrm{CPP}<45.5 \mathrm{~mm} \mathrm{Hg}$ presented $\mathrm{SD} / \mathrm{V} /$
Din $36 \%$ of the cases. One of the major reasons for the increased number of patients making a good recovery has been the early recognition and more aggressive treatment of disorders which can cause secondary brain injury. Insults of CPP $<50 \mathrm{~mm} \mathrm{Hg}$ were hardly tolerated, and ICP $>25 \mathrm{~mm} \mathrm{Hg}$ was associated with the poor outcome regardless of CPP. We want to emphasize that both surgeons and anesthesiologists must closely monitor BP throughout this early phase of care to maintain optimal CPP. 
A literature review shows that the main predictors of TBI surgery outcome are as follows: age; GCS on admission; pupil response and size; the presence of hypoxia; prolonged hypotension; hyperthermia; diffuse axonal injury or brain stem injury; characteristic CT features; and biomechanical parameters including ICP, CPP, and partial pressure of oxygen in brain tissue. ${ }^{21,22}$ The current study showed that ICP and CPP values obtained during surgery

Table 5 The demographic and clinical characteristics of the patients

\begin{tabular}{|l|l|}
\hline Variable & All patients $(\boldsymbol{n}=\mathbf{5 3})$ \\
\hline Age $(\mathrm{y})$ & $43.7 \pm 15.84$ \\
\hline Sex $(\mathrm{F} / \mathrm{M})$ & $13 / 40$ \\
\hline Mechanism & \\
\hline MVA & $79.2 \%$ \\
\hline Falls & $20.7 \%$ \\
\hline The severity of brain injury GCS & $5.39 \pm 1$ \\
\hline Type of hematoma & \\
\hline SDH & $34.1 \%$ \\
\hline CH & $22.6 \%$ \\
\hline Mixed & $43.3 \%$ \\
\hline GOS & \\
\hline Favorable & $64.1 \%$ \\
\hline Unfavorable & $35.8 \%$ \\
\hline
\end{tabular}

Abbreviations: $\mathrm{CH}$, chronic hematoma; GCS, Glasgow coma scale; GOS, Glasgow outcome scale; MVA, motor vehicle accident; SDH, subdural hematoma. for severe TBI are significantly associated with surgical outcomes. For predicting outcome and prognosis of surgery for severe TBI, we, therefore, recommend the use of ICP and CPP measurements taken either during surgery (i.e., after the creation of the first burr hole and after the evacuation of hematoma) and/or after surgery (i.e., after scalp closure). In this study, those with ICP $<28 \mathrm{~mm} \mathrm{Hg}$ or CPP $>45.5 \mathrm{~mm} \mathrm{Hg}$ after the first burr hole creation, those with ICP $<10.5 \mathrm{~mm} \mathrm{Hg}$ or CPP > $62 \mathrm{~mm} \mathrm{Hg}$ after hematoma evacuation, and ICP $<15.5 \mathrm{~mm} \mathrm{Hg}$ or CPP $>69.5 \mathrm{~mm} \mathrm{Hg}$ after wound closure had a significantly better prognosis and lower mortality compared with their counterparts. However, additional prospective, randomized, and controlled studies are needed to determine critical ICP and CPP thresholds after the first burr hole creation, after hematoma evacuation, and after scalp closure.

\section{Conclusion}

Monitoring ICP during surgery can improve outcomes of surgery for severe TBI by providing an early indication of increased ICP. Persons with severe TBI, initial ICP, and CPP provides great prognostic discrimination and is an independent predictor of surgical outcome. However, to provide a subjective guideline for managing severe TBI, further studies are needed to determine the optimal intraoperative thresholds for ICP and CPP.

Note

The Scientific Committee of APNSA had chosen my paper for the SVIMS Award Presentation in AP Neurocon- 2019 on 16 th June.

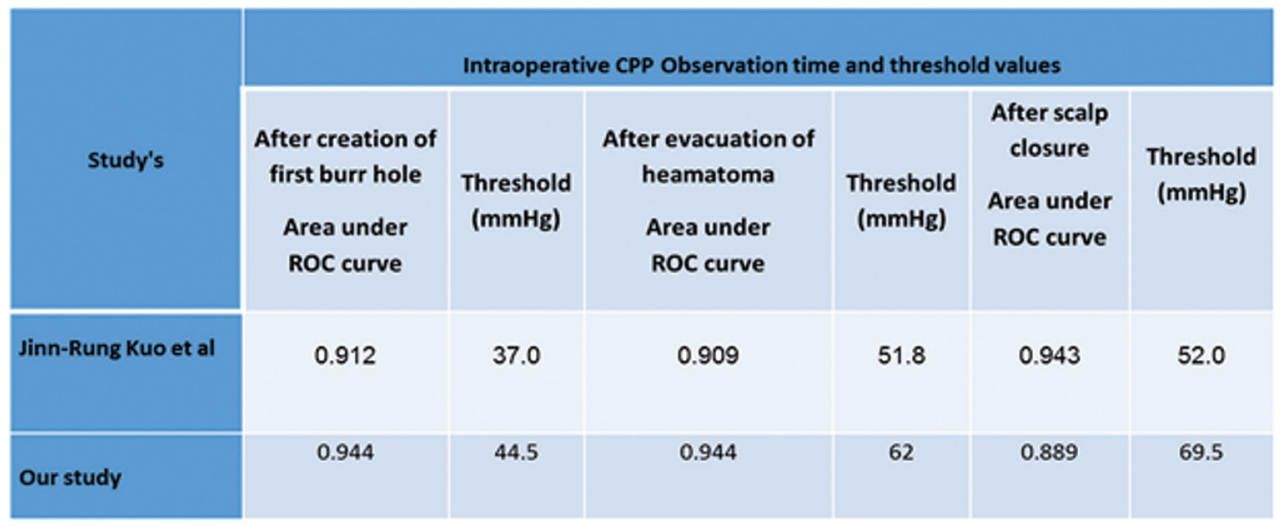

Fig. 7 Comparison of areas under ROC curves of CPP, ICP and cut off values with other study. CPP, cerebral perfusion pressure; ICP, intracranial pressure; ROC, receiver-operated characteristic. 


\section{Funding}

None.

\section{Conflict of Interest}

None declared.

\section{References}

1 Maas AIR, Stocchetti N, Bullock R. Moderate and severe traumatic brain injury in adults. Lancet Neurol 2008;7(8):728-741

2 Shen L, Wang Z, Su Z, et al. Effects of intracranial pressure monitoring on mortality in patients with severe traumatic brain injury: a meta-analysis. PLoS One 2016;11(12):e0168901

3 Olivecrona M, Rodling-Wahlström M, Naredi S, Koskinen LO. Effective ICP reduction by decompressive craniectomy in patients with severe traumatic brain injury treated by an ICPtargeted therapy. J Neurotrauma 2007;24(6):927-935

4 Cremer OL, van Dijk GW, van Wensen E, et al. Effect of intracranial pressure monitoring and targeted intensive care on functional outcome after severe head injury. Crit Care Med 2005;33(10):2207-2213

5 Lundberg N, Troupp H, Lorin H. Continuous recording of the ventricular-fluid pressure in patients with severe acute traumatic brain injury. A preliminary report. J Neurosurg 1965;22(6):581-590

6 Robertson CS, Valadka AB, Hannay HJ, et al. Prevention of secondary ischemic insults after severe head injury. Crit Care Med 1999;27(10):2086-2095

7 Stein SC, Georgoff P, Meghan S. Mirza KL, El Falaky OM. Relationship of aggressive monitoring and treatment to improved outcomes in severe traumatic brain injury. J Neurosurg 2010;112(5):1105-1112

8 Carney, NA. ,. Ghajar, J; Brain Trauma Foundation. American Association of Neurological SurgeonsCongress of Neurological SurgeonsJoint Section on Neurotrauma and Critical Care, AANS/CNS. J Neurotrauma 2007;24(Suppl 1) :S1-S106

9 Rosner MJ, Daughton S. Cerebral perfusion pressure management in head injury. J Trauma 1990;30(8):933-940, discussion 940-941

10 Verweij BH, Muizelaar JP, Vinas FC. Hyperacute measurement of intracranial pressure, cerebral perfusion pressure, jugular venous oxygen saturation, and laser Doppler flowmetry, before and during removal of traumatic acute subdural hematoma. J Neurosurg 2001;95(4):569-572

11 Jaeger M, Soehle M, Meixensberger J. Effects of decompressive craniectomy on brain tissue oxygen in patients with intracranial hypertension. J Neurol Neurosurg Psychiatry 2003;74(4):513-515

12 Schneider GH, Bardt $T$, Lanksch WR, Unterberg A. Decompressive craniectomy following traumatic brain injury: ICP, CPP and neurological outcome. Acta Neurochir Suppl (Wien) 2002;81:77-79

13 Kunze E, Meixensberger J, Janka M, Sörensen N, Roosen K. Decompressive craniectomy in patients with uncontrollable intracranial hypertension. Acta Neurochir Suppl (Wien) 1998;71:16-18

14 Huang SJ, Hong WC, Han YY, et al. Clinical outcome of severe head injury using three different ICP and CPP protocol-driven therapies. J Clin Neurosci 2006;13(8):818-822

15 Kuo J-R, Yeh T-C, Sung K-C, Wang CC, Chen CW, Chio CC. Intraoperative applications of intracranial pressure monitoring in patients with severe head injury. J Clin Neurosci 2006;13(2):218-223

16 Chambers IR, Treadwell L, Mendelow AD. Determination of threshold levels of cerebral perfusion pressure and intracranial pressure in severe head injury by using receiver-operating characteristic curves: an observational study in 291 patients. J Neurosurg 2001;94(3):412-416

17 Low D, Kuralmani V, Ng SK, Lee KK, Ng I, Ang BT. Prediction of outcome utilizing both physiological and biochemical parameters in severe head injury. J Neurotrauma 2009;26(8): $1177-1182$

18 Bratton SL, Chestnut RM, Ghajar J, et al; Brain Trauma FoundationAmerican Association of Neurological Surgeons Congress of Neurological Surgeonsjoint Section on Neurotrauma and Critical Care, AANS/CNS. Guidelines for the management of severe traumatic brain injury. VIII. Intracranial pressure thresholds. J Neurotrauma 2007;24(Suppl 1):S55-S58

19 Tsai TH, Huang TY, Kung SS, Su YF, Hwang SL, Lieu AS. Intraoperative intracranial pressure and cerebral perfusion pressure for predicting surgical outcome in severe traumatic brain injury. Kaohsiung J Med Sci 2013;29(10):540-546

20 Downard C, Hulka F, Mullins RJ, et al. Relationship of cerebral perfusion pressure and survival in pediatric brain-injured patients. J Trauma 2000;49(4):654-658, discussion 658-659

21 Jiang JY, Gao GY, Li WP, Yu MK, Zhu C. Early indicators of prognosis in 846 cases of severe traumatic brain injury. J Neurotrauma 2002;19(7):869-874

22 Wardlaw JM, Easton VJ, Statham P. Which CT features help predict outcome after head injury? J Neurol Neurosurg Psychiatry 2002;72(2):188-192, discussion 151 